Supporting Information

\title{
Highly Crystalline Films of Organic Small Molecules with Alkyl-Chains Fabricated by Weak Epitaxy Growth
}

Yangjie Zhu, ${ }^{\dagger}{ }^{\ddagger}$ Weiping Chen, ${ }^{\S}$ Tong Wang, ${ }^{\dagger}$ Haibo Wang, ${ }^{* \dagger}$ Yue Wang, ${ }^{\S}$ Donghang $\operatorname{Yan}^{\dagger}$

$\uparrow$ State Key Laboratory of Polymer Physics and Chemistry, Changchun Institute of Applied Chemistry, Chinese Academy of Sciences, Changchun 130022, P. R. China

\$ University of Chinese Academy of Sciences, Beijing 10039, P. R. China

$\S$ State Key Laboratory of Supramolecular Structure and Materials, College of Chemistry, Jilin University, Changchun 130012, P. R. China 


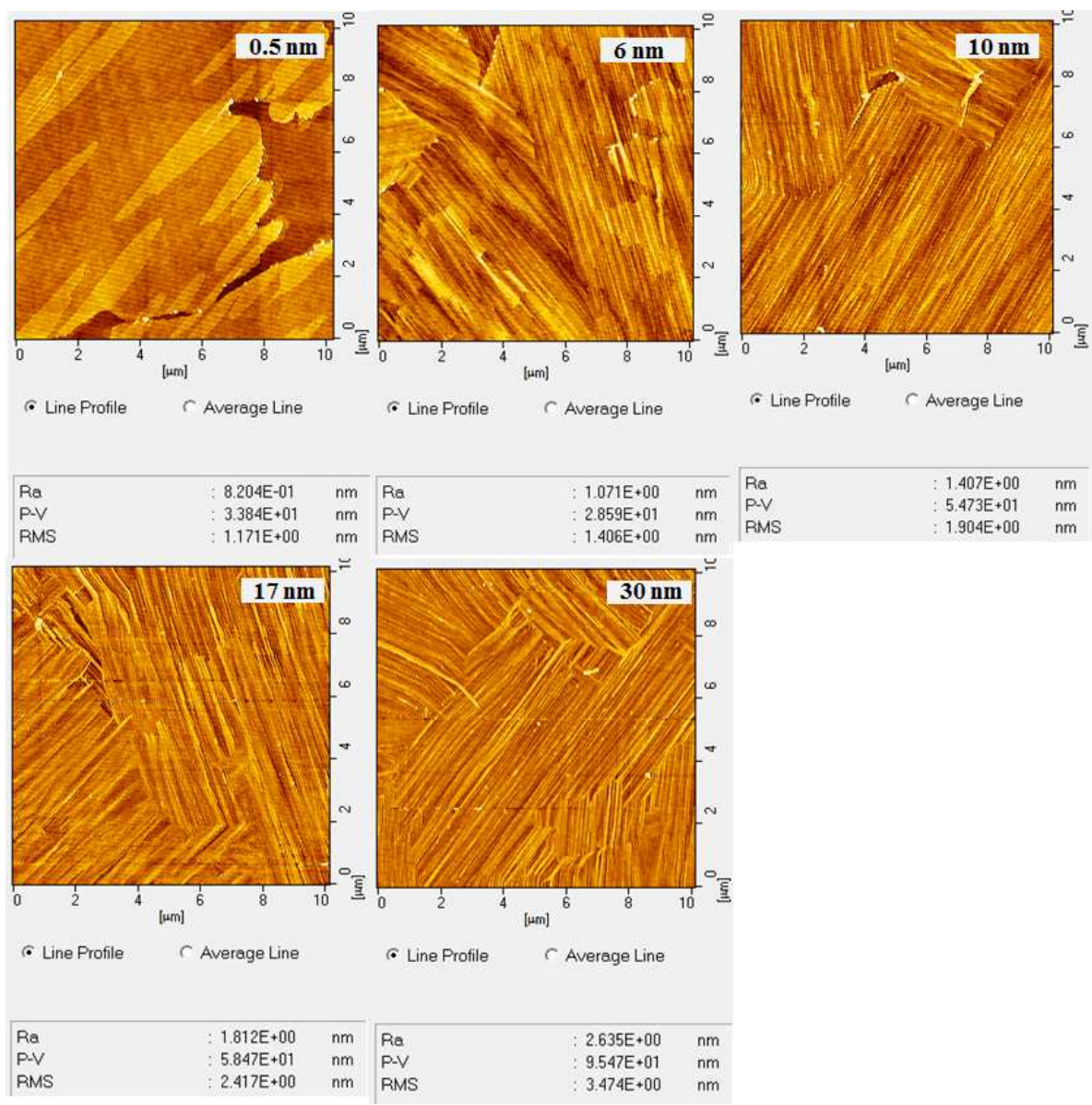

Fig. S1. The surface analysis of the C4-IDQA films in the Figure 2a, their corresponding surface roughness are $1.2 \mathrm{~nm}, 1.4 \mathrm{~nm}, 1.9 \mathrm{~nm}, 2.4 \mathrm{~nm}$ and $3.4 \mathrm{~nm}$. 


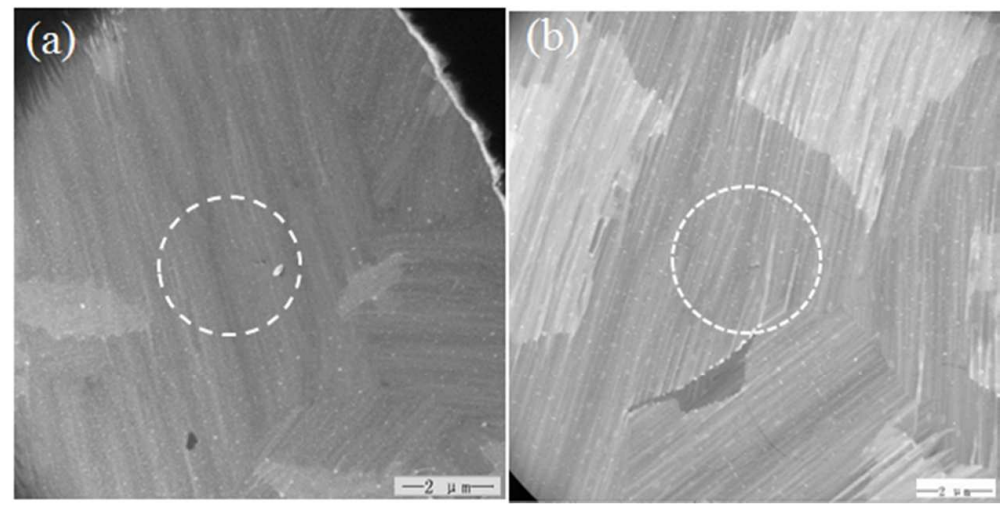

Fig. S2. The TEM dark field micrograph corresponding to Figure $2 \mathrm{~d}$ and e. 

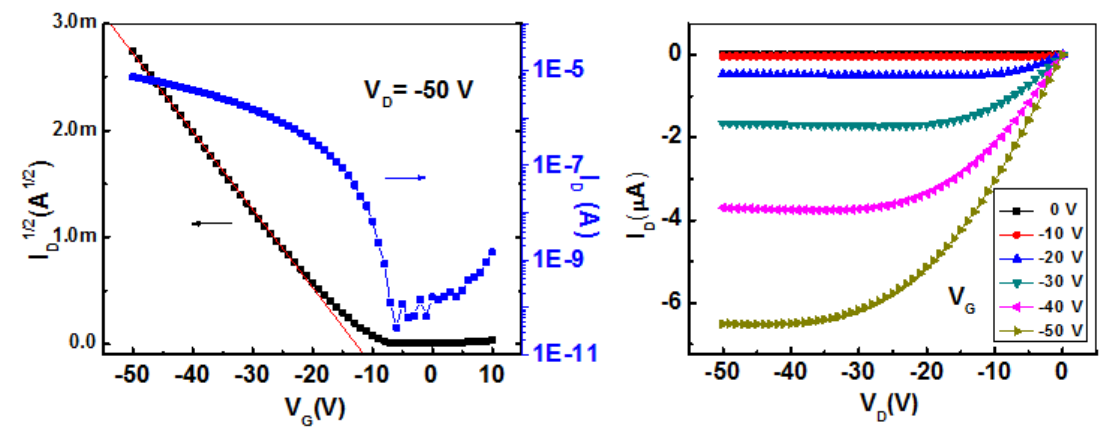

Fig. S3. Transfer and family curves from C4-IDQA OTFT based on $5 \mathrm{~nm} \mathrm{BP2T/30}$ nm C4-IDQA.
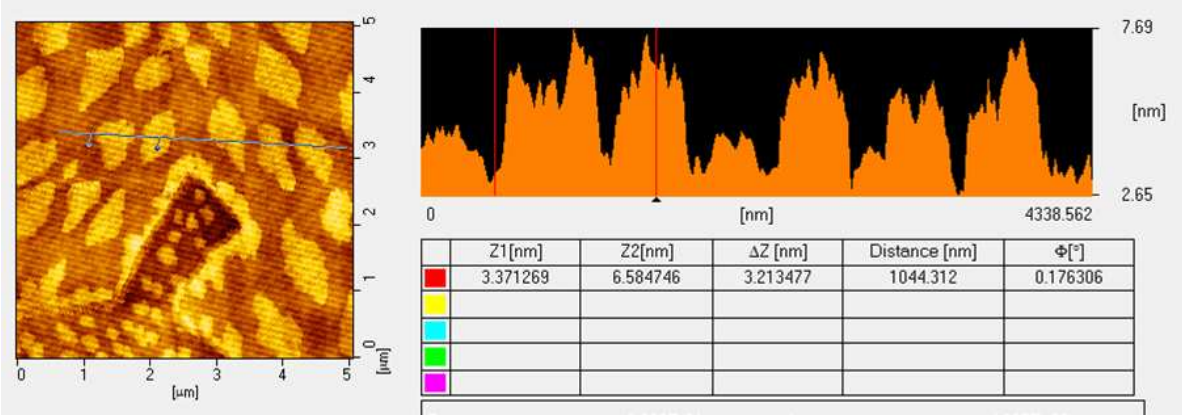

Fig. S4. The island height of $1 \mathrm{~nm}$ C4-IDQA grown at substrate temperature of 100 ${ }^{\circ} \mathrm{C}$. 


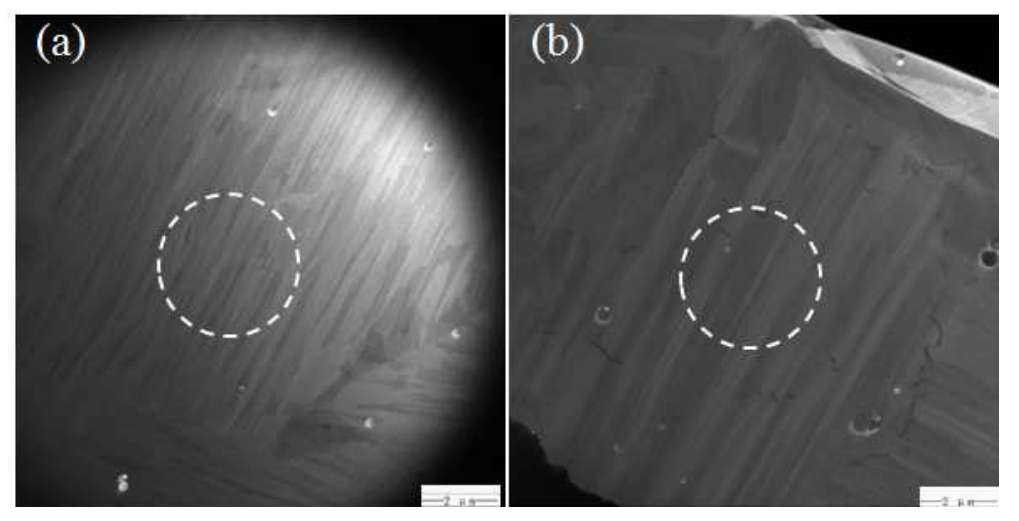

Fig. S5. The TEM dark field micrograph corresponding to Figure $6 \mathrm{~d}$ and e.
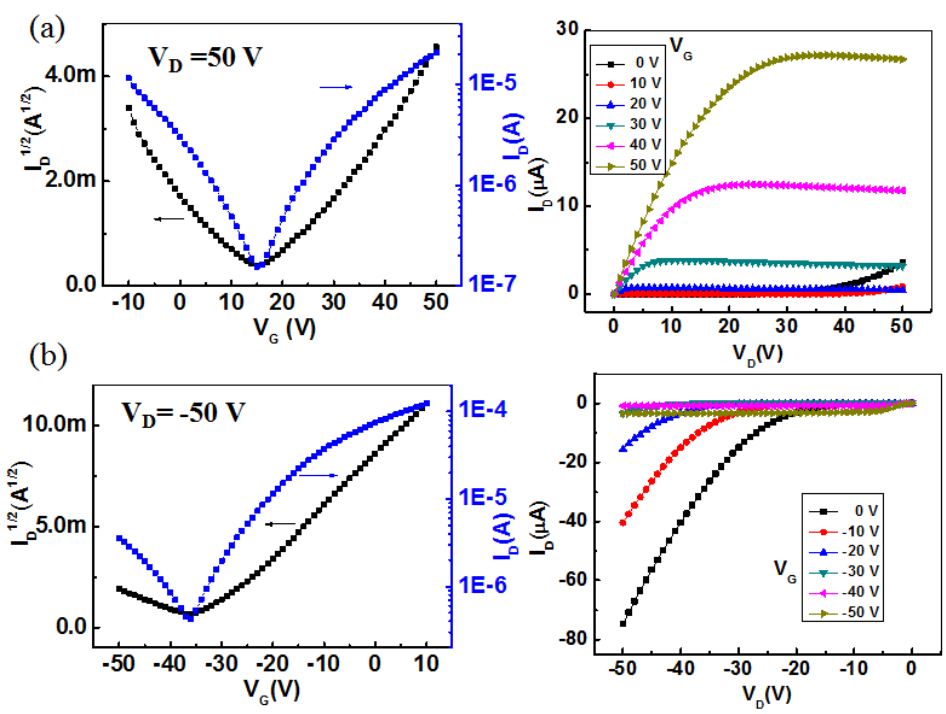

Fig. S6. The transfer and output curves of the OTFT based on $5 \mathrm{~nm} p-6 \mathrm{P} / 30 \mathrm{~nm}$ C8-PTCDI. (a) in the air; (b) the p-type transfer and output curves of the OTFT, the hole mobility is about $0.06 \mathrm{~cm}^{2} / \mathrm{Vs}$. 